\title{
New methods for the analysis of heartbeat behavior in risk stratification
}

\author{
Leon Glass $^{1}{ }^{*}$, Claudia Lerma $^{2}$ and Alvin Shrier ${ }^{1}$ \\ Department of Physiology, McGill University, Montreal, OC, Canada \\ 2 Departamento de Instrumentación Electromecánica, Instituto Nacional de Cardiologia "Ignacio Chávez," Tlalpan, Mexico
}

\section{Edited by:}

Heikki Veli Huikuri, University of Oulu,

Finland

Reviewed by:

Heikki Veli Huikuri, University of Oulu, Finland

Dipak K. D. Das, University of

Connecticut School of Medicine, USA

Celena Scheede-Bergdahl, McGill

University, Canada

*Correspondence:

Leon Glass, Department of

Physiology, McGill University, 3655

Promenade Sir William Osler,

Montreal, QC, Canada.

e-mail: leon.glass@mcgill.ca
Developing better methods for risk stratification for tachyarrhythmic sudden cardiac remains a major challenge for physicians and scientists. Since the transition from sinus rhythm to ventricular tachycardia/fibrillation happens by different mechanisms in different people, it is unrealistic to think that a single measure will be adequate to provide a good index for risk stratification. We analyze the dynamical properties of ventricular premature complexes over $24 \mathrm{~h}$ in an effort to understand the underlying mechanisms of ventricular arrhythmias and to better understand the arrhythmias that occur in individual patients. Two dimensional density plots, called heartprints, correlate characteristic features of the dynamics of premature ventricular complexes and the sinus rate. Heartprints show distinctive characteristics in individual patients. Based on a better understanding of the natures of transitions from sinus rhythm to sudden cardiac and the mechanisms of arrhythmia prior to cardiac arrest, it should be possible to develop better methods for risk stratification.

Keywords: cardiac arrhythmias, sudden cardiac death, ventricular tachycardia, non-linear dynamics, parasystole, early after depolarization

\section{INTRODUCTION}

Cardiac arrhythmias occur when the normal mechanisms of cardiac initiation and propagation no longer prevail and abnormal patterns of cardiac activity occur over some or all regions of the heart. Because of its clinical importance, the transition to tachyarrhythmic sudden cardiac death (for convenience, we use SCD here to indicate tachyarrhythmic sudden cardiac death) has attracted a large amount of attention. In particular, since tachyarrhythmias can generally be terminated by an implantable cardioverter-defibrillator (ICD), developing better means of predicting who will experience spontaneous transitions to tachyarrhythmias is a major focus for research. A large numbers of factors have been proposed that can be derived non-invasively from the electrocardiogram (ECG), but to date, none appear adequate (Huikuri et al., 2001; Goldberger et al., 2008, 2011). Many patients who would benefit from an ICD do not receive one, and many who receive one do not benefit. In view of the cost of ICDs and the potential complications of their use, the cost effectiveness of ICD use has been questioned (Tung et al., 2008).

A recent review identified several roadblocks to risk stratification (Goldberger et al., 2011). In the following we outline an approach that is complementary to current approaches. We argue that by relating the problem of risk stratification to basic science questions of dynamics and physiology, it should be possible to understand the physiology of individuals better and in this fashion develop better means for risk stratification.

We first briefly mention several risk factors that have been proposed. Then we describe an approach for analyzing arrhythmias in which there are frequent premature ventricular complexes (PVCs) that may help to identify the mechanisms generating those complexes. Finally, we consider the basic science question of analysis of the transition from sinus rhythm to tachycardia, and indicate how the various measures might be useful in helping to predict individuals at risk.

\section{RISK FACTORS FROM CLINICAL STUDIES}

Goldberger et al. (2008) provide an excellent survey of risk factors for SCD with extensive literature citations. We only provide selected references.

Variables that reflect parasympathetic and sympathetic activity play a prominent role in risk stratification for SCD (Barron and Lesh, 1996; La Rovere et al., 1998). In healthy hearts, there are typically wide fluctuations in the normal sinus rhythm over the course of the day. Various measures have been used to document reduction of these fluctuations by analyzing the SDs of the fluctuations in time, the power spectra of the heart rate, and other statistical measures derived from non-linear dynamics (Voss et al., 2009). The heart rate turbulence, reflecting the fluctuations in the sinus rhythm following a PVC, is also a reflection of parasympatheticsympathetic function (Schmidt et al., 1999). Since less healthy hearts display lower levels of fluctuations of the sinus rate many of these measures are an indirect reflection of ventricular function. Higher levels of sympathetic activity may be pro-arrhythmogenic since they might predispose the heart to PVCs arising from early after depolarizations (EADs; Zipes, 1991) or other mechanisms.

A second class of risk factors for SCD relate directly to cardiac anatomy and physiology. The most important of these is the left ventricular ejection fraction (LVEF), where low LVEF $(<30$ $35 \%$ ) is often used to identify candidates for an ICD (Bardy, 2005). Another important risk factor reflecting cardiac physiology is inducibility of ventricular tachycardia (VT) using programmed electrical stimulation, since a positive test indicates an anatomical 
substrate for VT (Vandepol et al., 1980; Wellens et al., 1985; Josephson, 2008). Another test that has attracted great attention recently is T-wave alternans, where an elevated level of alternation of Twaves at higher pacing rates or sinus rates reflects higher risk (Rosenbaum et al., 1994; Koller et al., 1998; Sato et al., 2006; Qu et al., 2010). Additional risk factors include an increased width of terminal portions of the QRS complex on the signaled-averaged ECG (Gomes et al., 2001), greater frequency of PVCs (Carrim and Khan, 2005), and long QT intervals (Zipes, 1991; Fermini and Fossa, 2003) all of which confer a higher risk of SCD.

\section{THE HEARTPRINT AND MECHANISMS FOR PVCs}

From the previous analysis of risk factors for SCD, it is clear that we have not yet identified a single factor - or even combination of factors - that are adequate for risk stratification for SCD. The large number of factors investigated to date, all of which are associated with an increased risk, suggest that there may be many potential mechanisms for transitions from sinus rate to tachyarrhythmias. A given factor would be expected to be useful as a predictor for a certain mechanism but not others. Consequently, we believe that an important step in the development of better risk stratification markers for SCD will be to develop methods that are useful for identifying specific physiological mechanisms in the individual that might be underlying arrhythmias (Glass and Lerma, 2006).

In order to do this, we have proposed a method, called the heartprint to capture dynamical features of ventricular premature ventricular complexes (PVCs) in Holter recording as a function of sinus period (NN) see Figures $\mathbf{1}$ and $\mathbf{2}$ (Schulte-Frohlinde et al., 2002). Heartprints give a visual display of the qualitative and quantitative features of the dynamics over the entire $24-\mathrm{h}$ period. A heartprint is a way to represent dependencies between the $\mathrm{NN}$ interval and (i) the ectopic beat interval (between two $\mathrm{V}$ beats, or VV interval), (ii) the number of intervening beats (NIB) between two consecutive PVCs, and (iii) the coupling interval (CI) from a sinus beat to the next PVC. The ordinate of the 3 grayscale plots in the heartprint is the NN interval. The incidence of the VV intervals, $\mathrm{NIB}$ values, and the $\mathrm{CI}$ are indicated in the grayscale plots, respectively, where the relative frequency of occurrence is indicated by the shading, (e.g., black is associated with the highest incidence). The plots above the grayscale plots give the histograms of the $\mathrm{VV}$ intervals, the NIB values, and the CI, respectively. The histogram to the left of the grayscale plots gives the histogram of $\mathrm{NN}$ values.
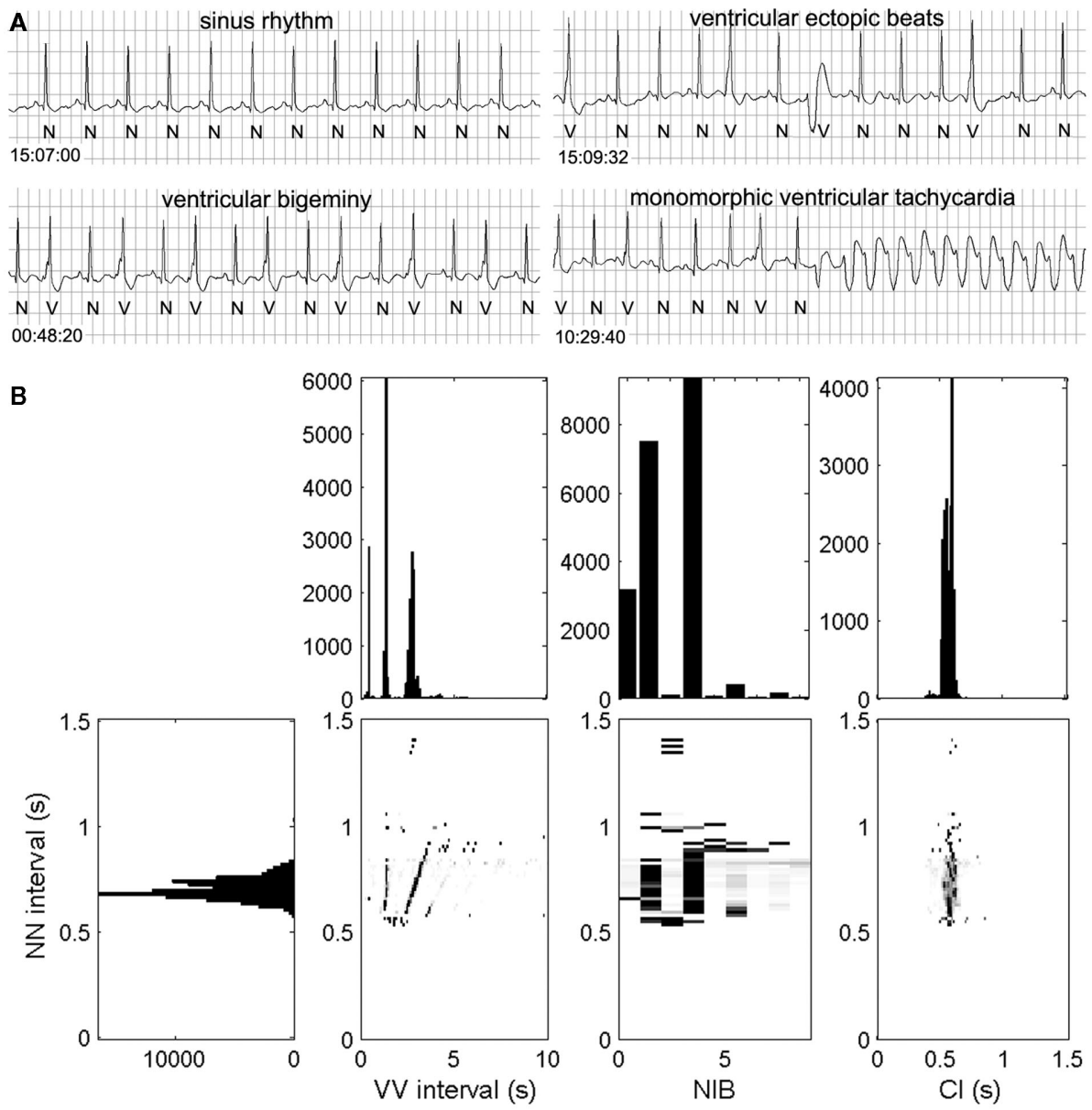

FIGURE 1 | Rhythm strips (A) and heartprint (B) for a male patient with unknown clinical history. Heartprint shows sinus rates with NN intervals between 0.5 and $1 \mathrm{~s}$, the number of intervening beats between two ectopic

beats (NIB) were mostly 0, 1 and 3, and the coupling interval $(\mathrm{Cl})$ was relatively fixed. Case 44 from the PhysioNet Sudden Cardiac Death Data Base (Goldberger et al., 2000). For abbreviations, see text. 

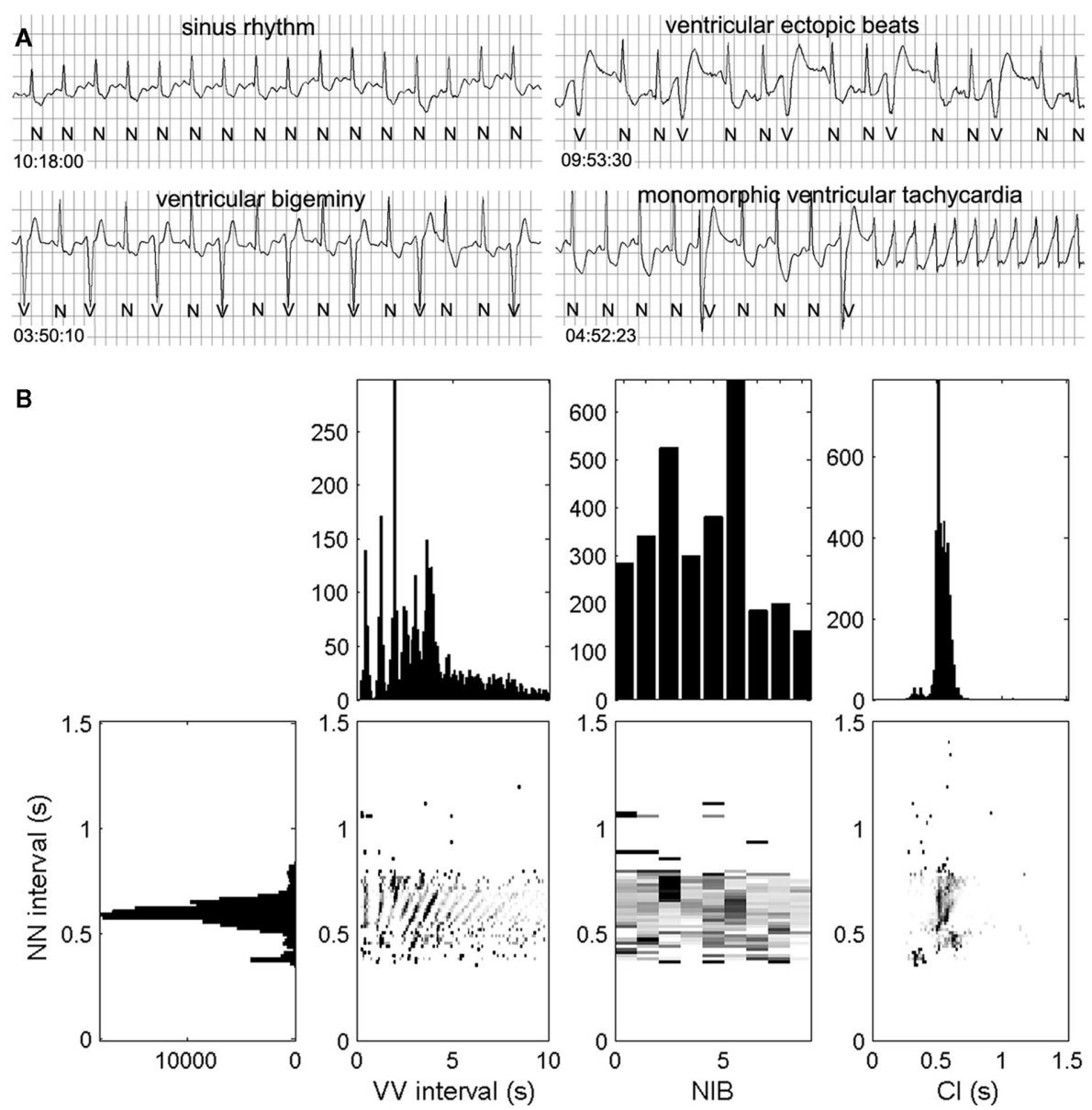

FIGURE 2 | Rhythm strip (A) and the heartprint (B) for an 80-year-old male patient with unknown clinical history. Heartprint shows that NN intervals were between 0.4 and $0.8 \mathrm{~s}$ with a wide range of NIB values and coupling interval (CI) was highly variable. Case 48 from the PhysioNet Sudden Cardiac Death Data Base (Goldberger et al., 2000).

In Figures 1 and 2, we show ECG traces and heartprints for two different patients from the PhysioNet Sudden Cardiac Death Database (Goldberger et al., 2000). The dynamical features of the arrhythmias are very different, potentially implying that different mechanisms may be underlying the arrhythmia in each patients. If the mechanisms of the PVCs are independent of the mechanism for the transition to tachycardia, then understanding the mechanisms of the PVCs might be an interesting academic exercise, but it would be of little practical importance. But in the current records, the presence of the PVCs immediately prior to the tachycardia, may reflect an underlying causal relationship.

In previous work we have found some striking patterns of PVC occurrence that reflect on the mechanism. In one set of patients with long QT syndrome, the CIs were strongly peaked and there were frequent bigeminal episodes in which there is an alternation between sinus beats and PVCs (Lerma et al., 2007). This triad of features is consistent with long QT syndrome, and might of itself be a novel marker for SCD. Record 44 shares some of these characteristics, but has a confounding feature of two morphologies of PVCs. Another different striking set of characteristics found in some patients are variable CIs, but strong dependence of the pattern of NIBs as a function of the sinus rate, consistent with theoretical predictions based on a mechanism in which there is an independent ectopic (parasystolic) ventricular focus (Schulte-Frohlinde et al., 2002).

Although in a limited number of circumstances, such as the two mentioned above, we can propose physiological mechanisms that are consistent with the features of the heartprint, in most of the heartprint records that we have examined, we do not yet know how to decode the underlying mechanisms. Despite a significant literature on the interpretation of complex arrhythmias based on comparatively short rhythm strips (Pick and Langendorf, 1979), there is a need for developing better ways of identifying mechanisms of arrhythmia based on Holter tape or other long term data.

\section{THE TRANSITION TO VT}

We view the transition to VT as a problem both in clinical medicine and in basic science. Our perspective derives from the mathematical field of non-linear dynamics (Guckenheimer and Holmes, 1983). Non-linear dynamics focuses on qualitative features of dynamics. The classification of different types of cardiac 
arrhythmias by clinicians combined with the development of mathematical models for dynamics and transitions observed in certain arrhythmias such as atrio-ventricular heart block fully support the relevance of non-linear dynamics to understand mechanisms and dynamics in the heart (Glass et al., 1987a,b). Although classification of transitions between sinus rhythm and tachycardias is not yet nearly as developed, there have been a large number of proposals, many enriched by the interplay between clinical medicine and basic science. We briefly summarize several of these mechanisms.

(i) Induction of monomorphic VT by programmed electrical stimulation. A classic electrophysiologic approach to risk stratification involves the induction of monomorphic VT by delivery of 1-3 premature stimuli in patients with an anatomically based reentrant pathway (Vandepol et al., 1980; Wellens et al., 1985; Josephson, 2008). The successful induction of VT with the same morphology as observed in an ambulatory ECG can provide an anatomical target for ablation. Outside of the electrophysiology laboratory, the VT might be induced by a PVC generated by some other mechanism. This type of arrhythmia is common in patients with a prior myocardial infarction. It can be hemodynamically stable with a well defined exit point for the reentrant pathway into the ventricle. Thus, it can potentially be detected and treated. Although in principle the reentrant pathway could also lead to isolated PVCs, it is not clear how to identify this mechanism for PVCs.

(ii) Induction of reentrant tachycardia associated with spiral waves by delivery of a single pulse in the vulnerable period. The classical concept of PVCs falling on the T-wave of the electrocardiogram ( $\mathrm{R}$ on $\mathrm{T}$ ) has a counterpart in the theoretical and experimental studies that demonstrate the initiation of spiral waves by the delivery of a stimulus during the repolarization phase of a propagating action potential (Witkowski et al., 1998). In the heart, a PVC could be generated by some physiologic mechanism. In the theoretical literature, there is often the assumption that the rotating spiral would be associated with monomorphic VT, but it seems likely to us, that in a heterogeneous heart the spiral might drift or even break up, leading to a polymorphic VT or fibrillation.

(iii) VT induced by an accelerated ectopic focus. One of the classic examples is slow monomorphic VT originating from the right ventricular outflow tract (Belhassen, 2005). This is typically benign, even though it might be associated with a large number of PVCs per day. Typically the coupling intervals of PVCs to the preceding sinus beat are long. However, variants have been described in which the coupling intervals are shorter, and may be associated with initiation of dangerous polymorphic VT (Viskin and Antzelevitch, 2005).

(iv) Initiation of ventricular tachycardia by break up of propagating waves in the context of alternating action potential duration (T-wave alternans). The identification of alternation of action potential duration in experimental and mathematical models has a rich mathematical development from analysis of the action potential restitution curve (Guevara et al., 1984; Koller et al., 1998; Sato et al., 2006; Qu et al., 2010). In spatially heterogeneous systems, action potentials with a short duration might be locally blocked leading to wave break and initiation of reentrant arrhythmias. Fenton et al. (2002) have documented several different ways in which regularly propagating spiral waves in mathematical models of cardiac tissue in which alternans is prominent can become unstable leading to reentrant dynamics similar to those that are believed to underlie ventricular fibrillation.

(v) Initiation of polymorphic VT in the setting of genetic abnormalities or drug effects that lead to long QT syndrome and EADs. It is well known that a long QT interval can arise as a consequence of genetic abnormalities in potassium channels (Sanguinetti and Tristani-Firouzi, 2006) or as an unwanted side effect of medication (Fermini and Fossa, 2003) and is an important risk factor for sudden death. Recent theoretical and experimental studies (Sato et al., 2009; Auerbach et al., 2011) have documented the way in which EADs can be generated and lead to reentrant tachycardia. Experimental studies of arrhythmias induced by potassium channel $\left(\mathrm{I}_{k r}\right)$ blocking drugs show induction of couplets or triplets that could stand as an experimental model of arrhythmogenic sites (Kim et al., 2009).

(vi) Initiation of reentry in hearts with heterogeneity and reduced coupling. Fibrosis or sarcoidosis is sometimes found on autopsy of subjects with sudden cardiac death (Fabre and Sheppard, 2006). These clinical observations have a counterpart in experimental and theoretical models of heart cells in tissue culture demonstrating the spontaneous initiation of reentrant arrhythmias in situations of increased heterogeneity and reduced coupling (Bub et al., 1998, 2002).

This listing is not meant to be exhaustive but rather illustrative. Although listed separately, more than one mechanism might operate together in an individual patient. For example, a monomorphic reentrant tachycardia associated with anatomical reentry could degenerate into ventricular fibrillation as a consequence of heterogeneity and/or blockage secondary to alternans. Further, although we list induction of VT by R on T and EADs separately, EADs may be simply be one mechanism for generating $\mathrm{R}$ on $\mathrm{T}$.

\section{ANALYSIS OF ARRHYTHMIA AND DYNAMICS IN MODEL SYSTEMS AND IN INDIVIDUAL PATIENTS}

We believe that the above discussion provides a foundation and suggests a strategy for developing better methods for risk stratification based on a better understanding of the individual physiology, combined with a better understanding of the mechanisms of transition to tachyarrhythmias. What is necessary is to develop better methods for characterization of physiological mechanisms of arrhythmia in patients and to correlate that with outcome data.

In order to make progress we suggest three parallel research paths.

\section{MATHEMATICAL ANALYSIS OF TRANSITION TO TACHYCARDIA IN MODEL SYSTEMS}

Experimental studies in model cardiac systems have demonstrated a variety of circumstances that lead to spontaneous transition to reentrant rhythms including variation of the coupling between cells (Bub et al., 2002), addition of drugs that modify ionic 
channels leading to long action potential durations and EADs (Sato et al., 2009), modifications of geometry leading to heterogeneities (Auerbach et al., 2011), addition of drugs that lead to alternation of action potential duration, and instabilities during rapid propagation (Qu et al., 2010). As is clear, this is a very active and rich area for basic science research. As our ability to modify geometry and physiology in tissue culture improves, there should continue to be strong progress.

\section{CLASSIFICATION OF ARRHYTHMIAS IN PEOPLE BASED ON HOLTER RECORDS AND ADDITIONAL CLINICAL DATA}

Electrocardiographic data from the human heart can be readily collected and constitute a vast store of data that remains incompletely understood. From an examination of the records in Figures $\mathbf{1}$ and 2, it is clear that individuals have very different characteristics, and that standard methods for PVC arrhythmia analysis which rely largely on counting frequencies of PVCs do not address fine details that reflect the mechanism (Carrim and Khan, 2005). Yet, since the Cardiac Arrhythmia Suppression Trial it has been clear that a simple consideration and manipulation of the frequency of PVCs is not adequate (Echt et al., 1991). Developing deeper insight into the classification of mechanisms of arrhythmia remains a challenge for the future.

\section{MODELING OF THE ARRHYTHMIAS IN INDIVIDUAL PATIENTS}

Several different styles of model can provide insight into arrhythmia mechanisms. Simplified models of parasystole can be used to explain subtle dynamical details of the occurrence of PVCs in selected patients (Moe et al., 1977; Schulte-Frohlinde et al., 2001, 2002). The essential feature is to carry out quantitative models over extended periods of time (e.g., hours), taking into account the possibility that cardiac parameters may change as a consequence of activity, drugs, or other environmental factors. A complementary

\section{REFERENCES}

Aguado-Sierra, J., Krishnamurthy, A., Villongco, C., Chuang, J., Howard, E., Gonzales, M. J., Omens, J., Krummen, D. E., Narayan, S., Kerckhoffs, R. C., and McCulloch, A. D. (2011). Patient-specific modeling of dyssynchronous heart failure: a case study. Prog. Biophys. Mol. Biol. (in press). doi:10. 1016/j. pbiomolbio. 2011. 06. 014

Auerbach, D. S., Grzda, K. R., Furspan, P. B., Sato, P. Y., Mironov, S., and Jalife, J. (2011). Structural heterogeneity promotes triggered activity, reflection and arrhythmogenesis in cardiomyocyte monolayers. J. Physiol. (Lond.) 589, 2363-2381.

Bardy, G. H. (2005). Sudden cardiac death in heart failure trial (SCDHeFT) investigators: amiodarone or an implantable cardioverterdefibrillator for congestive heart failure. N. Engl. J. Med. 352, 225-237.

Barron, H. V., and Lesh, M. D. (1996). Autonomic nervous system and

style of analysis involves detailed simulations based on realistic anatomical data (Aguado-Sierra et al., 2011). Although modeling realistic anatomies is still extremely difficult, there is rapid development of imaging methods and computer power. Yet, development of realistic models based on clinical data that are capable of predictive value for susceptibility to arrhythmia and transition to VT still seems to be remote. However, collaborations between basic scientists, engineers, and clinicians in this direction remains an important future direction.

\section{CONCLUSION}

In their recent review, Goldberger et al. (2011) raised and rejected the possibility that "better risk stratification for SCD is unachievable." Although the problem is clearly difficult, we have argued that progress should be possible by adopting a complementary approach to risk stratification compared to current clinical approaches. Based on the notion that there are multiple routes for the transition from sinus rhythm to tachyarrhythmic SCD, we would not expect a single risk factor to be adequate. Even taking multiple factors, each of which might be useful for different individuals, would not be adequate to predict risk. However, an absence of those factors, might be a useful predictor of low risk (as has been found; Goldberger et al., 2011).

We argue for the analysis of mechanisms of arrhythmias in individual patients as a strategy to better develop methods of risk stratification. Combination of such a classic approach (Pick and Langendorf, 1979) with modern methods of computer analysis and simulation may yet provide insight into disease as well as new practical methods for risk stratification.

\section{ACKNOWLEDGMENTS}

We thank the Canadian Heart and Stroke Foundation and MPRIME (formerly MITACS) for financial support.

(1991). Mortality and morbidity in patients receiving encainide, flecainide, or placebo. N. Engl. J. Med. 324, 781-788.

Fabre, A., and Sheppard, M. N. (2006). Sudden adult death syndrome and other non-ischaemic causes of sudden cardiac death. Heart 92, 316-320.

Fenton, F. H., Cherry, E. M., Hastings, H. M., and Evans, S. J. (2002). Multiple mechanisms of spiral wave breakup in a model of cardiac electrical activity. Chaos 12, 852-892.

Fermini, B., and Fossa, A. A. (2003). The impact of drug-induced QT interval prolongation on drug discovery and development. Nat. Rev. Drug Discov. 2, 439-447.

Glass, L., Guevara, M. R., and Shrier, A. (1987a). Universal bifurcations and the classification of cardiac arrhythmias. Ann. N. Y. Acad. Sci. 504, 168-178.

Glass, L., Goldberger, A. L., Courtemanche, M., and Shrier, A. (1987b). Nonlinear dynamics, chaos and complex cardiac arrhythmias. Proc. R. Soc. Lond. A. Math. Phys. Sci. 413, 9-26.

Glass, L., and Lerma, C. (2006). Risk stratification for arrhythmic sudden cardiac death. Heart Rhythm 3, 1497-1501.

Goldberger, A. L., Amaral, L. A. N., Glass, L., Hausdorff, J. M., Ivanov, P. C., Mark, R. G., Mietus, J. E., Moody, G. B., Peng, C. K., and Stanley, H. E. (2000). Physiobank, physiotoolkit, and physionet: components of a new research resource for complex physiologic signals. Circulation 101, e215e220.

Goldberger, J. J., Buxton, A. E., Cain, M., Costantini, O., Exner, D. V., Knight, B. P., Lloyd-Jones, D., Kadish, A. H., Lee, B., Moss, A., Myerburg, R., Olgin, J., Passman, R., Rosenbaum, D., Stevenson, W., Zareba, W., and Zipes, D. P. (2011). Risk stratification for arrhythmic sudden cardiac death: identifying the roadblocks. Circulation 123 , 2423-2430. 
Goldberger, J. J., Cain, M. E., Hohnloser, S. H., Kadish, A. H., Knight, B. P., Lauer, M. S., Maron, B. J., Page, R. L., Passman, R. S., Siscovick, D., Siscovick, D., Stevenson, W. G., Zipes, D. P., American Heart Association, American College of Cardiology Foundation, and Heart Rhythm Society. (2008). American Heart Association/American College of Cardiology Foundation/Heart Rhythm Society scientific statement on noninvasive risk stratification techniques for identifying patients at risk for sudden cardiac death. Circulation 118, 1497-1518.

Gomes, J. A., Cain, M. E., Buxton, A. E., Josephson, M. E., Lee, K. L., and Hafley, G. E. (2001). Prediction of long-term outcomes by signal-averaged electrocardiography in patients with unsustained ventricular tachycardia, coronary artery disease, and left ventricular dysfunction. Circulation 104, 436-441.

Guckenheimer, J., and Holmes, P. (1983). Nonlinear Oscillations, Dynamical Systems, and Bifurcations of Vector Fields. New York, NY: Springer-Verlag.

Guevara, M., Ward, G., Shrier, A., and Glass, L. (1984). Electrical alternans and period doubling bifurcations. IEEE Comp. Cardiol. 562, 167-170.

Huikuri, H. V., Castellanos, A., and Myerburg, R. J. (2001). Sudden death due to cardiac arrhythmias. $N$. Engl. J. Med. 345, 1473-1482.

Josephson, M. E. (2008). Clinical Cardiac Electrophysiology: Techniques and Interpretations. Philadelphia, PA: Lippincott Williams \& Wilkins.

Kim, M. Y., Aguilar, M., Hodge, A., Vigmond, E., Shrier, A., and Glass, L. (2009). Stochastic and spatial influences on drug-induced bifurcations in cardiac tissue culture. Phys. Rev. Lett. 103, 58101.
Koller, M. L., Riccio, M. L., and Gilmour, R. L. Jr. (1998). Dynamic restitution of action potential duration during electrical alternans and ventricular fibrillation. Am. J. Physiol. Heart Circ. Physiol. 275, H1635-H1642.

La Rovere, M. T., Bigger, J. T. Jr., Marcus, F. I., Mortara, A., and Schwartz, P. J. (1998). Baroreflex sensitivity and heart-rate variability in prediction of total cardiac mortality after myocardial infarction. ATRAMI (autonomic tone and reflexes after myocardial infarction) investigators. Lancet 351, 478-484.

Lerma, C., Lee, C. F., Glass, L., and Goldberger, A. L. (2007). The rule of bigeminy revisited: analysis in sudden cardiac death syndrome. $J$. Electrocardiol. 40, 78-88.

Moe, G. K., Jalife, J., Mueller, W. J., and Moe, B. (1977). A mathematical model of parasystole and its application to clinical arrhythmias. Circulation 56, 968-969.

Pick, A., and Langendorf, R. (1979). Interpretation of Complex Arrhythmias. Philadelphia: Lea \& Febiger.

Qu, Z., Xie, Y., Garfinkel, A., and Weiss, J. N. (2010). T-wave alternans and arrhythmogenesis in cardiac diseases. Front. Physiol. 1:154. doi:10.3389/fphys.2010.00154

Rosenbaum, D. S., Jackson, L. E., Smith, J. M., Garan, H., Ruskin, J. N., and Cohen, R. J. (1994). Electrical alternans and vulnerability to ventricular arrhythmias. N. Engl. J. Med. 330, 235-241.

Sanguinetti, M. C., and Tristani-Firouzi, M. (2006). hERG potassium channels and cardiac arrhythmia. Nature 440, 463-469.

Sato, D., Shiferaw, Y., Garfinkel, A., Weiss, J. N., Qu, Z., and Karma, A. (2006). Spatially discordant alternans in cardiac tissue. Role of calcium cycling. Circ. Res. 99, 520-527.
Sato, D., Xie, L. H., Sovari, A. A., Tran, D. X., Morita, N., Xie, F., Karagueuzian, H., Garfinkel, A., Weiss, J. N., and Qu, Z. (2009). Synchronization of chaotic early after depolarizations in the genesis of cardiac arrhythmias. Proc. Natl. Acad. Sci. U.S.A. 106, 2983-2988.

Schmidt, G., Malik, M., Barthel, P., Schneider, R., Ulm, K., Rolnitzky, L., Camm, A. J., Bigger, J. T. Jr., and Schömig, A. (1999). Heart-rate turbulence after ventricular premature beats as a predictor of mortality after acute myocardial infarction. Lancet 353, 1390-1396.

Schulte-Frohlinde, V., Ashkenazy, Y., Goldberger, A. L., Ivanov, P. C., Costa, M., Morley-Davies, A., Stanley, H. E., and Glass, L. (2002). Complex patterns of abnormal heartbeats. Phys. Rev. E Stat. Nonlin. Soft Matter Phys. 66, 031901.

Schulte-Frohlinde, V., Ashkenazy, Y., Ivanov, P. C., Glass, L., Goldberger, A. L., and Stanley, H. E. (2001). Noise effects on the complex patterns of abnormal heartbeats. Phys. Rev. Lett. 87,68104 .

Tung, R., Zimetbaum, P., and Josephson, M. E. (2008). A critical appraisal of implantable cardioverterdefibrillator therapy for the prevention of sudden cardiac death. J. Am. Coll. Cardiol. 52, 1111-1121.

Vandepol, C. J., Farshidi, A., Spielman, S. R., Greenspan, A. M., Horowitz, L. N., and Josephson, M. E. (1980). Incidence and clinical significance of induced ventricular tachycardia. Am. J. Cardiol. 45, 725-731.

Viskin, S., and Antzelevitch, C. (2005). The cardiologists' worst nightmare: sudden death from "benign" ventricular arrhythmias. J. Am. Coll. Cardiol. 46, 1295-1297.

Voss, A., Schulz, S., Schroeder, R. Baumert, M., and Caminal, P.
(2009). Methods derived from nonlinear dynamics for analysing heart rate variability. Philos. Trans. A Math. Phys. Eng. Sci. 367, 277-296.

Wellens, H. J., Brugada, P., and Stevenson, W. G. (1985). Programmed electrical stimulation of the heart in patients with life-threatening ventricular arrhythmias: what is the significance of induced arrhythmias and what is the correct stimulation protocol? Circulation 72, 1-7.

Witkowski, F. X., Leon, L. J., Penkoske, P. A., Giles, W. R., Spano, M. L., Ditto, W. L., and Winfree, A. T. (1998). Spatiotemporal evolution of ventricular fibrillation. Nature 392, 78-82.

Zipes, D. P. (1991). The long QT interval syndrome. A Rosetta stone for sympathetic related ventricular tachyarrhythmias. Circulation 84 , 1414-1419.

Conflict of Interest Statement: Leon Glass is a recipient of research funds and licensing agreements with Medtronic.

Received: 28 September 2011; accepted: 09 November 2011; published online: 29 November 2011.

Citation: Glass L, Lerma $C$ and Shrier A (2011) New methods for the analysis of heartbeat behavior in risk stratification. Front. Physio. 2:88. doi: 10.3389/fphys.2011.00088

This article was submitted to Frontiers in Clinical and Translational Physiology, a specialty of Frontiers in Physiology. Copyright (c) 2011 Glass, Lerma and Shrier. This is an open-access article distributed under the terms of the Creative Commons Attribution Non Commercial License, which permits use, distribution, and reproduction in other forums, provided the original authors and source are credited. 